BMJ Open

Diabetes

Research

\& Care

\title{
Association of socioeconomic status and DKA readmission in adults with type 1 diabetes: analysis of the US National Readmission Database
}

\author{
Estelle Everett, ${ }^{\circledR}$ Nestoras Nicolas Mathioudakis ${ }^{\circledR}$
}

To cite: Everett E, Mathioudakis NN. Association of socioeconomic status and DKA readmission in adults with type 1 diabetes: analysis of the US National Readmission Database. BMJ Open Diab Res Care 2019;7:e000621. doi:10.1136/ bmjdrc-2018-000621

Received 27 September 2018 Revised 11 March 2019 Accepted 15 March 2019

\section{Check for updates}

(C) Author(s) (or their employer(s)) 2019. Re-use permitted under CC BY-NC. No commercial re-use. See rights and permissions. Published by BMJ.

Endocrinology, Johns Hopkins University, Baltimore, Maryland, USA

Correspondence to Dr Nestoras Nicolas Mathioudakis; nmathio1@jhmi.edu

\section{ABSTRACT}

Objective To identify patient and hospital predictors of recurrent diabetic ketoacidosis (DKA) admissions in adults in the USA with type 1 diabetes, focusing on socioeconomic indicators.

Research design and methods This cross-sectional study used the National Readmission Database to identify adult patients with type 1 diabetes admitted for DKA between 2010 and 2015. The index DKA admission was defined as the first admission within the calendar year and the primary outcome was recurrent DKA admission(s) within the same calendar year. Multivariable logistic regression analysis was performed using covariates of patient and hospital factors at the index admission to determine the odds of DKA readmission(s).

Results Among 181284 index DKA admissions, 39693 (22\%) had at least one readmission within the calendar year, of which 33931 (86\%) and 5762 (14\%) had 1-3 and $\geq 4$ DKA readmissions, respectively. When compared with the highest income quartile, patients in the first and second income quartiles had $46 \%(95 \% \mathrm{Cl} 30 \%$ to $64 \%)$ and $34 \%(95 \% \mathrm{Cl} 19 \%$ to $51 \%)$ higher odds of four or more DKA readmissions, respectively. Medicaid and Medicare insurance were both associated with a 3.3-fold adjusted risk ( $95 \% \mathrm{Cl} 3.0$ to 3.7 ) for $\geq 4$ readmissions compared with private insurance, respectively. Younger age, female sex, and discharge against medical advice were also predictive.

Conclusions Lower socioeconomic status and Medicaid insurance are strong predictors of DKA readmissions in adults with type 1 diabetes in the USA. Further studies are needed to understand the mediators of this association to inform multilevel interventions for this high-risk population. Significance of the study The association of socioeconomic status (SES) and hospital admission for DKA has been studied in pediatrics with type 1 diabetes, but the data in adults are limited, and studies evaluating recurrent DKA admissions are scarcer. To our knowledge, this is the first study to describe predictors of recurrent DKA admissions in adults with type 1 diabetes on a national level in the USA. We found that those at highest risk of recurrent DKA are young women with low SES who had Medicaid or Medicare insurance. These findings should prompt further studies to explore the mediators of these disparities in patients with type 1 diabetes, as recurrent DKA results in high healthcare utilization and increased risk of long-term complications.

\section{Significance of this study}

What is already known about this subject?

- Diabetic ketoacidosis (DKA) is a serious acute complication of type 1 diabetes (T1D) that is associated with poor clinical outcomes.

- Studies evaluating the association of socioeconomic status and recurrent DKA are limited in adult populations of T1D, especially in the USA.

What are the new findings?

- In this nationally representative sample of adults with T1D, we found that being from the lowest income quartile was associated with a near $50 \%$ increased odds of $>4$ DKA readmissions in a calendar year.

- Other factors associated with recurrent DKA admission included younger age, female sex, Medicaid, Medicare, no insurance, and discharge against medical advice.

How might these results change the focus of research or clinical practice?

- Further research is needed to understand the drivers of these disparities so that we can intervene in this high-risk population.

\section{INTRODUCTION}

Diabetic ketoacidosis (DKA) is a serious acute complication of type 1 diabetes and the leading cause of death in children and young adults in this population. A study in the UK found that while a single episode of DKA has been associated with a $5.2 \%$ risk of death, that risk increases by 6 -fold with five or more admissions. ${ }^{1}$ In the USA, despite recent advances in medical care of type 1 diabetes (eg, closed-loop insulin pumps, continuous glucose monitoring), the number of hospitalizations for DKA in 2009 had increased by $40 \%$ from the previous decade. ${ }^{2}$ Medical expenditures related to these DKA hospitalizations are estimated to be over US\$ 2.4 billion. $^{3}$ 
Inferences regarding the socioeconomic factors linked to readmission for DKA among adults in the USA are primarily derived from limited studies conducted in children with type 1 diabetes ${ }^{4-8}$ or adults with type 1 diabetes in European countries. ${ }^{2910}$ Most of the studies conducted in the USA were primarily restricted to single hospitals, hospital systems or registries, limiting their generalizability. ${ }^{2} 11$ The Type 1 Diabetes Exchange Registry evaluated the role of socioeconomic factors on admission for DKA, but this study focused on predictors of at least one admission as the investigators could not reliably measure the number of DKA admissions for each patient, which were largely self-reported. ${ }^{12}$ Although there have been studies exploring predictors of hospital readmission in adults with type 2 diabetes, these studies evaluated all-cause admissions, not DKA admissions specifically. ${ }^{13}$

Several measures are considered independent surrogates of socioeconomic status (SES), including education, occupation, income, and area level measures. ${ }^{14}$ Area level measures are especially good indicators of SES in the USA as social structures often segregate persons by SES, thus making where one resides a very useful indicator of their SES. ${ }^{14}$ Using the National Readmission Database, which strategically samples US hospitals to allow generalizability to the entire country, we sought to evaluate the role of SES and other patient and hospital factors on DKA readmission in adults with type 1 diabetes in the USA. The primary objective of this study was to determine whether known area level median income, a well-established proxy for SES, is independently associated with readmission for DKA. We hypothesized that lower median income would be associated with higher likelihood of readmission. A secondary objective was to explore whether other patient and hospital factors, some of which are also established social determinants of health, are associated with this outcome. Despite the higher risk of DKA for adults with type 1 diabetes compared with other diabetes types, to our knowledge no previous study has focused specifically on identifying predictors of DKA readmission on a national level in this patient population.

\section{RESEARCH DESIGN AND METHODS Study population}

This study used the Healthcare Cost and Utilization Project National Readmission Database (NRD), developed by the US Agency for Healthcare Research and Quality. The NRD is a publicly available deidentified database containing admission data from hospitals in 27 geographically dispersed states, accounting for $58 \%$ of the total US resident population and $57 \%$ of all US hospitalizations. We selected all patients aged 18 or older with an admission diagnosis of type 1 diabetes who had at least one admission for DKA between January 1, 2010 and December 31, 2015. Patient admissions were identified through International Classification of Disease (ICD)-9 codes reported in the NRD as a primary diagnosis, defined by the NRD as the condition at the time of discharge thought to be chiefly responsible for the admission to the hospital. We used four ICD-9 codes (250.11, 250.13, 250.21, and 250.23), which code for either ketoacidosis or hyperosmolarity in either controlled or uncontrolled type 1 diabetes. Hyperosmolarity ICD-9 codes (250.21 and 250.23) accounted for the primary diagnosis for only $2 \%$ of admissions. These admissions were included in the study as approximately $40 \%$ of participants that had an admission with a primary admission diagnosis of hyperosmolality also had either a secondary diagnosis of DKA or had other admissions with a primary diagnosis was DKA. There was also a subset of admissions with a primary DKA diagnosis but also had a secondary diagnosis of hyperosmolality. Considering the prevalence of mixed DKA with hyperosmolality in type 1 diabetes ${ }^{15-19}$ and the lack of convention for coding these patients, ${ }^{15}$ the very low number of patients $(\sim 1 \%)$ with only hyperosmolality ICD codes, were considered to have negligible influence on the generalizability of the findings to patients with type 1 diabetes and DKA.

\section{Predictor variables}

NRD variables are reported by admission and include hospital-level and patient-level data. Given that we had an interest in characteristics that predict readmission, the covariates analyzed in this study represent characteristics from the index admission, which we defined as the first hospitalization for DKA in the calendar year.

Patient-level variables included age, sex, urban-rural classification, whether the patient resided in the same state in which they were hospitalized, median income, admission payer, length of stay (LOS), number of chronic illnesses (defined as conditions lasting 12 months or longer requiring ongoing medical intervention or selfcare), and number of diagnoses. Race and ethnicity data are not available in the NRD. Number of diagnoses and chronic diseases were determined by ICD-9 codes on file at the time of discharge. Residential status was reported per the 2010 United States urban-rural classification, which defines an urbanized area as 50000 residents or more and rural as less than 50000 residents. Median household income was reported as quartiles by the NRD and was established based on median income data per zip code for the calendar year. Admission payer included Medicare, Medicaid, private payers (private health maintenance organizations or prefeered provider organization), and other (worker's compensation, title V, Civilian Health and Medical Health and Medical Program of the Department of Veterans Affairs or other government programs).

Hospital-level variables included rural-urban classification, size, ownership, and teaching status. Hospital ownership was described as either government, private/ non-profit, and private/investment. A teaching hospital was defined as having an American Medical Associationapproved residency program, by being a member of the Council of Teaching Hospitals or having a ratio of fulltime equivalent interns and residents to beds of 0.25 or 
higher. The size of a hospital was characterized as small, medium, or large, based on several factors including bed size adjusting for US region, rural or urban location, and teaching status. Other predictor variables included were weekday versus weekend admission, LOS, and disposition location.

\section{Outcome variable}

The primary outcome was readmission for DKA occurring after but within the same calendar year as the index admission. Since each admission receives a unique identifier that is consistent at the patient level for a calendar year and there is no patient-specific identifier across calendar years, admissions could only be tracked for a given patient within a calendar year and a given patient could have multiple index admissions in different calendar years. Consequently, the follow-up time interval was variable depending on the time of year of the index admission (ie, only 1 month of follow-up data available for an admission occurring on December 1, 2015 vs 6 months of follow-up data for an admission occurring on July 1, 2015).

We evaluated DKA readmissions as a categorical outcome with three levels: no readmission, 1-3 readmissions, and 4 or more readmissions. The rationale for selecting these categories was based on a previous study that demonstrated a substantial increase in mortality for patients with 5 or more admissions (ie, 4 or more readmissions).

\section{Statistical analysis}

Descriptive statistics were used to characterize the overall study population and by DKA readmission outcome category at the level of patient admissions. Since all continuous variables were non-normally distributed (as assessed by the Skewness and Kurtosis test), medians and IQRs are reported and counts and frequencies are reported for categorical data. Statistical differences were calculated for each of the readmission groups relative to the no readmission group. For continuous measures, the Wilcoxon rank-sum test was used to analyze differences given the non-parametric distribution. The $\chi^{2}$ test was used to evaluate differences in proportions of categorical variables.

Multivariable logistic regression was used to evaluate the association of DKA readmission categories and predictor variables. Given the large sample size of the study, we expected most predictors to be statistically significant even if the magnitude of effect was small; to select the most parsimonious model, we relied on the best subsets achieved by Aikaike information criterion rather than stepwise regression. ${ }^{20} \mathrm{~A}$ variance inflation factor $<10$ was considered to indicate collinearity, ${ }^{21}$ and no variables were found to be collinear. The patient's urban-rural status was not included in the model due to a large proportion of missing data. Other than this variable, there was near complete ascertainment in the NRD for most predictors. The following variables had very little $(<2 \%)$ missing data: payer, median income, patient urban/rural status, and LOS. Considering the nearly complete outcome ascertainment and lack of significant differences in the proportion of missing data by outcome group, we excluded admissions with any missing data from the regression analyses.

Two regression models were built with respect to the DKA outcome: $1-3$ readmissions (model 1 ) and $\geq 4$ readmissions (model 2), with the reference group being no readmissions for each model. For model 1 , the following variables were included: age, sex, median income, insurance type, whether patient is a resident of the state in which they were hospitalized, number of chronic illnesses, number of diagnoses, hospital bed size, LOS, and disposition. Model 2 additionally contained hospital urban/rural status as a covariate.

Discharge-level weights provided by the NRD were used to inflate effect estimates to national estimates, accounting for the stratified single-stage cluster sampling design. All analyses were performed with Stata statistical software, V.14.2 (StataCorp). A two-sided p value of $<0.05$ was considered statistically significant.

\section{RESULTS}

Among 264948 DKA admissions of patients with type 1 diabetes, 181284 were identified as index admissions. Among these 181284 index DKA admissions, 39693 (22\%) had at least one readmission, of which 33931 $(86 \%)$ and $5762(14 \%)$ had $1-3$ and $\geq 4$ DKA readmissions, respectively, in the calendar year. Overall, in a calendar year, the total number of DKA readmissions ranged from 1 to 19 . In the 1-3 readmission group, the median time to the first readmission was 73 days. Approximately $25 \%$ had their first readmission within 30 days, $43 \%$ within 60 days, and $58 \%$ within 90 days. In the $\geq 4$ readmission group, the median time to the first readmission was 33 days, with $47 \%, 72 \%$, and $86 \%$ of the first readmissions occurring within 30,60 , and 90 days, respectively.

Baseline characteristics of the entire cohort and readmission subgroups are reported in table 1 .

Overall, this study cohort consisted of admissions of relatively young (median age 35 ) patients with type 1 diabetes from low-income areas $(62 \%$ below the 50 th percentile for median income) admitted to large (58\%), urban $(88 \%)$, private $(84 \%)$, non-teaching $(88 \%)$ hospitals. When comparing the two groups with readmissions with the group without readmissions, we observed several differences. An inverse association was noted with age and women were disproportionally represented in the DKA readmission groups. There was an inverse association with median income and category of DKA readmissions: the proportion of patients in the lowest income quartile increased with increasing DKA admission category. The proportion of admissions covered by private insurance declined with increasing DKA readmission category, with nearly $50 \%$ fewer admissions covered by private insurance in those with $\geq 4$ DKA readmissions compared with 
Table 1 Baseline characteristics at index admission

\begin{tabular}{|c|c|c|c|c|c|c|}
\hline & Entire cohort & DKA readmissi & ns & & $P$ value & \\
\hline & & None & $1-3$ & $\geq 4$ & $\begin{array}{l}1-3 \text { vs } \\
\text { None }\end{array}$ & $\begin{array}{l}\geq 4 \text { vs } \\
\text { None }\end{array}$ \\
\hline Age, years & $35(25,49)$ & $37(26,51)$ & $30(23,42)$ & $26(22,35)$ & $<0.001$ & $<0.001$ \\
\hline Sex, N (\%) & & & & & $<0.001$ & $<0.001$ \\
\hline Rural urban status, N (\%) & & & & & 0.031 & 0.028 \\
\hline Urban & 67223 (83.9) & 53012 (84.1) & 12185 (83.4) & 2026 (82.4) & & \\
\hline Rural & 12904 (16.1) & 10038 (15.9) & 2434 (16.6) & $432(17.6)$ & & \\
\hline In-state residency†, N (\%) & & & & & $<0.001$ & $<0.001$ \\
\hline Quartile 1 & 63359 (35.6) & 48379 (34.8) & 12626 (37.8) & 2354 (41.6) & & \\
\hline Quartile 2 & $46482(26.1)$ & $35951(25.8)$ & 9004 (27.0) & $1527(27.0)$ & & \\
\hline Quartile 3 & 40523 (22.7) & 31949 (23.0) & $7384(22.1)$ & 1190 (21.0) & & \\
\hline Quartile 4 & $27813(15.6)$ & $22837(16.4)$ & $4390(13.1)$ & $586(10.4)$ & & \\
\hline Payer, N (\%) & & & & & $<0.001$ & $<0.001$ \\
\hline Medicare & 33526 (18.5) & 26765 (19.0) & 5895 (17.4) & $866(15.0)$ & & \\
\hline Medicaid & 47847 (26.5) & 33741 (23.9) & $11542(34.1)$ & $2564(44.7)$ & & \\
\hline Private & $55188(30.5)$ & $46618(33.0)$ & 7667 (22.7) & $903(15.7)$ & & \\
\hline Weekend admission, $\mathrm{N}(\%)$ & $48651(26.8)$ & $37778(26.7)$ & $9234(27.2)$ & $1639(28.4)$ & 0.047 & 0.003 \\
\hline LOS, days & $2(2,4)$ & $2(2,4)$ & $2(2,4)$ & $2(2,4)$ & $<0.001$ & $<0.001$ \\
\hline Disposition, N (\%) & & & & & $<0.001$ & $<0.001$ \\
\hline Routine & 155062 (85.6) & $120824(85.4)$ & 29327 (86.5) & 4911 (85.3) & & \\
\hline Hospital transfer & $772(0.4)$ & $643(0.5)$ & $122(0.4)$ & $7(0.1)$ & & \\
\hline Facility transfer & 5903 (3.3) & $5129(3.6)$ & $708(2.1)$ & $66(1.2)$ & & \\
\hline Home health & $9634(5.3)$ & $8051(5.7)$ & $1348(4.0)$ & $235(4.1)$ & & \\
\hline AMA & $9043(5.0)$ & $6097(4.3)$ & $2405(7.0)$ & $541(9.3)$ & & \\
\hline Death & $756(0.4)$ & $756(0.5)$ & $0(0.0)$ & $0(0.0)$ & & \\
\hline Unknown & $24(0.01)$ & $18(0.01)$ & $6(0.02)$ & $0(0.0)$ & & \\
\hline $\begin{array}{l}\text { Hospital rural urban status, N } \\
(\%)\end{array}$ & & & & & $<0.001$ & 0.001 \\
\hline Rural & 22177 (12.2) & $17063(12.0)$ & 4335 (12.8) & $779(13.5)$ & & \\
\hline Urban & 159107 (87.8) & $124528(88.0)$ & 29596 (87.2) & $4983(86.5)$ & & \\
\hline Hospital size, N (\%) & & & & & 0.251 & 0.667 \\
\hline Small & $24581(13.6)$ & 19159 (13.5) & 4666 (13.8) & $756(13.1)$ & & \\
\hline Medium & 52341 (28.9) & 40807 (28.8) & 9869 (29.1) & 1665 (29.0) & & \\
\hline
\end{tabular}


Table 1 Continued

\begin{tabular}{|c|c|c|c|c|c|c|}
\hline & \multirow[t]{2}{*}{ Entire cohort } & \multicolumn{3}{|c|}{ DKA readmissions } & \multicolumn{2}{|c|}{ P value* } \\
\hline & & None & $1-3$ & $\geq 4$ & $\begin{array}{l}1-3 \text { vs } \\
\text { None }\end{array}$ & $\begin{array}{l}\geq 4 \text { vs } \\
\text { None }\end{array}$ \\
\hline Large & $104362(57.6)$ & $81625(57.7)$ & 19396 (57.2) & 3341 (57.9) & & \\
\hline Ownership, N (\%) & & & & & 0.060 & 0.668 \\
\hline Government & $29100(16.0)$ & $22572(15.9)$ & $5584(16.4)$ & $944(16.4)$ & & \\
\hline Private/non-profit & 120141 (66.3) & $93986(66.4)$ & 22349 (65.9) & $3806(66.0)$ & & \\
\hline Private/investment & $32043(17.7)$ & $25033(17.7)$ & $5998(17.7)$ & $1012(17.6)$ & & \\
\hline Teaching hospital, N (\%) & $22177(12.2)$ & $17063(12.1)$ & $4335(12.8)$ & $779(13.5)$ & $<0.001$ & 0.001 \\
\hline
\end{tabular}

*Wilcoxon signed-rank test for comparison of continuous variables and $\chi^{2}$ for categorical variables.

†Hospital in same state as patient's state of residence. For continuous variables (age, no of chronic illnesses, no of diagnoses, no of admissions, and LOS), medians and IQRs are reported.

AMA, against medical advice; DKA, diabetic ketoacidosis; LOS, length of stay.

those with none. Similarly, the proportion of admissions covered by Medicaid increased by $60 \%$ in the highest DKA readmission group. There were no notable differences observed in number of chronic illnesses and number of diagnoses, median length of stay, weekday versus weekend admission, hospital size, and hospital ownership. Following discharge, transfer to another hospital/facility or home health services was associated with lower prevalence of DKA readmission. Conversely, leaving the hospital against medical advice (AMA) was associated with higher prevalence of DKA readmission. The proportions of patients admitted to teaching hospitals increased with DKA readmission categories.

The results of the fully adjusted regression models are shown in figure 1 and table 2.

With respect to SES, there was a $19 \%$ and $46 \%$ increase, respectively, in the adjusted odds of $1-3$ and $\geq 4$ DKA

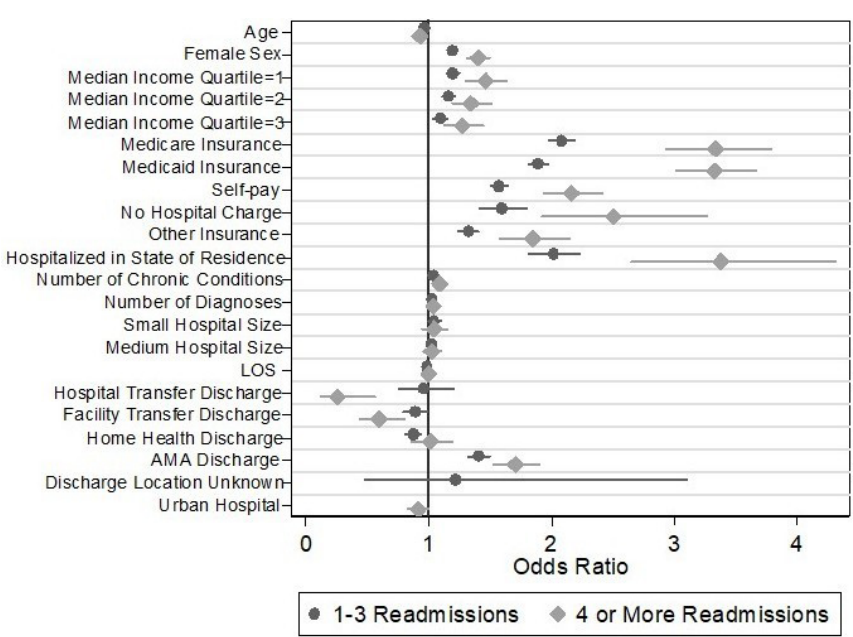

Figure 1 Risk factors for diabetic ketoacidosis (DKA) readmissions comparing those without DKA readmissions with those with 1-3 and $\geq 4$, respectively. Reference for categorical variables are fourth income quartile for median income, private insurance for payer, large hospital size, routine discharge for type of discharge. AMA, against medical advice; LOS, length of stay. readmissions for those in the lowest income quartile compared with those in the highest. With respect to insurance status, the adjusted odds of readmission was 2.08 (95\% CI 1.97 to 2.19 ) and 3.33 (95\% CI 2.94 to 4.379 ) for those with Medicare in the $1-3$ and $\geq 4$ DKA readmissions, respectively, compared with those with private insurance. For Medicaid patients, these odds were 1.89 (95\% CI 1.82 to 1.97 ) and 3.33 (95\% CI 3.02 to 3.67 ), respectively. After adjustment, increasing age was associated with lower odds of DKA readmission, with increasing effect size by higher DKA readmission category. Women had a significantly higher odds of DKA readmission, with a $40 \%$ increase in odds of $\geq 4$ readmissions.

Although length of stay was not associated with DKA readmission, hospital transfer, facility transfer, or discharge with home health services each reduced the odds of 1-3 readmissions by approximately $10 \%-15 \%$, with increasing effect size in the higher DKA readmission category. Conversely, patients who were discharged against medical advice had approximately a $41 \%$ increased odds of 1-3 admissions and 70\% increased odds for $\geq 4$ readmissions. Hospital size, hospital teaching status, and hospital ownership were only weakly associated with DKA readmission.

\section{DISCUSSION}

In this study, we found that lower SES, using the surrogate of median area income, was strongly associated with DKA readmission. Similarly, insurance status, an established social determinant of health that is closely linked to SES, was the strongest predictor of DKA readmission. To place these findings in context, a patient from an area with the lowest income quartile would be expected to have a $46 \%$ increase in the odds of four or more DKA readmissions in a given calendar year, while a patient with Medicare insurance would have over a 3-fold increased odds of this outcome.

The inverse association with median income and DKA readmission in this study expands on previous 
Table 2 Predictors of DKA readmission in multivariable logistic regression models

\begin{tabular}{|c|c|c|c|c|c|c|}
\hline \multirow[b]{2}{*}{ Variable } & \multicolumn{3}{|c|}{$1-3$ vs No DKA readmissions } & \multicolumn{3}{|c|}{$\geq 4$ vs No DKA readmissions } \\
\hline & OR & $(95 \% \mathrm{Cl})$ & $P$ value & OR & $(95 \% \mathrm{Cl})$ & $P$ value \\
\hline Age & 0.96 & 0.96 to 0.96 & $<0.001$ & 0.93 & 0.92 to 0.93 & $<0.001$ \\
\hline \multicolumn{7}{|l|}{ Sex } \\
\hline Female & 1.19 & 1.16 to 1.23 & $<0.001$ & 1.40 & 1.32 to 1.49 & $<0.001$ \\
\hline Male & 1.00 & (ref) & - & 1.00 & (ref) & - \\
\hline \multicolumn{7}{|l|}{ In-state residence } \\
\hline Non-resident & 1.00 & (ref) & - & 1.00 & (ref) & - \\
\hline Resident & 2.02 & 1.82 to 2.23 & $<0.001$ & 3.38 & 2.6 to 4.31 & $<0.001$ \\
\hline \multicolumn{7}{|l|}{ Median income quartile } \\
\hline Quartile 1 & 1.19 & 1.14 to 1.25 & $<0.001$ & 1.46 & 1.30 to 1.64 & $<0.001$ \\
\hline Quartile 2 & 1.16 & 1.11 to 1.22 & $<0.001$ & 1.34 & 1.19 to 1.51 & $<0.001$ \\
\hline Quartile 3 & 1.09 & 1.04 to 1.15 & $<0.001$ & 1.28 & 1.13 to 1.44 & $<0.001$ \\
\hline Quartile 4 & 1.00 & (ref) & - & 1.00 & (ref) & $<0.001$ \\
\hline \multicolumn{7}{|l|}{ Payer } \\
\hline Medicare & 2.08 & 1.97 to 2.19 & $<0.001$ & 3.33 & 2.94 to 3.79 & $<0.001$ \\
\hline Medicaid & 1.89 & 1.82 to 1.97 & $<0.001$ & 3.33 & 3.02 to 3.67 & $<0.001$ \\
\hline Private & 1.00 & (ref) & - & 1.00 & (ref) & - \\
\hline Self-pay & 1.57 & 1.50 to 1.65 & $<0.001$ & 2.16 & 1.94 to 2.41 & $<0.001$ \\
\hline No charge & 1.59 & 1.41 to 1.80 & $<0.001$ & 2.50 & 1.92 to 3.27 & $<0.001$ \\
\hline Other & 1.32 & 1.24 to 1.41 & $<0.001$ & 1.85 & 1.58 to 2.15 & $<0.001$ \\
\hline No of chronic illnesses & 1.04 & 1.03 to 1.05 & $<0.001$ & 1.09 & 1.06 to 1.11 & $<0.001$ \\
\hline No of diagnoses & 1.03 & 1.03 to 1.05 & $<0.001$ & 1.03 & 1.02 to 1.05 & $<0.001$ \\
\hline LOS & 0.99 & 0.98 to 0.99 & $<0.001$ & 1.00 & 0.99 to 1.01 & 0.673 \\
\hline \multicolumn{7}{|l|}{ Disposition } \\
\hline Routine & 1.00 & (ref) & - & 1.00 & (ref) & - \\
\hline Hospital transfer & 0.95 & 0.76 to 1.20 & 0.691 & 0.26 & 0.12 to 0.57 & 0.001 \\
\hline Facility transfer & 0.89 & 0.80 to 0.99 & 0.028 & 0.59 & 0.44 to 0.80 & 0.001 \\
\hline Home health & 0.87 & 0.81 to 0.94 & $<0.001$ & 1.01 & 0.86 to 1.19 & 0.881 \\
\hline AMA & 1.41 & 1.33 to 1.49 & $<0.001$ & 1.70 & 1.53 to 1.91 & $<0.001$ \\
\hline Unknown & 1.22 & 0.48 to 3.10 & 0.68 & - & - & $<0.001$ \\
\hline \multicolumn{7}{|l|}{ Hospital size } \\
\hline Small & 1.05 & 1.00 to 1.09 & 0.06 & 1.05 & 0.95 to 1.15 & 0.372 \\
\hline Medium & 1.02 & 0.99 to 1.06 & 0.17 & 1.03 & 0.95 to 1.10 & 0.493 \\
\hline Large & 1.00 & (ref) & - & 1.00 & (ref) & - \\
\hline \multicolumn{7}{|c|}{ Hospital rural urban status } \\
\hline Rural & - & - & - & 1.0 & (ref) & - \\
\hline Urban & - & - & - & 0.02 & 0.02 to 0.03 & 0.053 \\
\hline
\end{tabular}

AMA, against medical advice; DKA, diabetic ketoacidosis; LOS, length of stay; ref, reference.

findings from the Type 1 Diabetes Exchange Registry, which showed nearly 2-fold increased odds of at least one DKA admission in those with a household income of US\$35 000 or less compared with those with US $\$ 75$ 000 or more. ${ }^{12}$ Unlike that study, which was conducted in an ambulatory population with type 1 diabetes where the outcome was a single DKA admission (most of which were patient reported), our study was focused on confirmed DKA readmissions, which represents a higher risk outcome. Additionally, in that study, extremes of income were evaluated, while the present study used a greater number of income categories, which are normalized to the general US population over time and allow inferences to be drawn regarding the magnitude and strength of the association. Our findings also align with those obtained in a UK population which showed 
a 2-fold risk of DKA admission in patients from more deprived areas. ${ }^{10}$

With respect to insurance status, only one other study has evaluated the association of DKA readmission and payer in adults with diabetes; however, this study included a mix of adult patients with type 1 and type 2 diabetes limited to the Chicago area and only reported proportions of patients with different payers without any adjusted measure of association. In that study, among patients with 2-3 DKA admissions, 19\% were insured by Medicaid and $22 \%$ by Medicare, while only $18 \%$ had private insurance. In comparison, among the equivalent group in our study (1-3 readmissions), 34\% were insured by Medicaid, $17 \%$ by Medicare, and $23 \%$ by private insurance. Thus, compared with the Chicago study, there was a much larger proportion of patients insured by Medicaid nationally who had a moderate number of DKA readmissions.

In the type 1 diabetes population (Type 1 Diabetes Exchange Registry), non-private insurance status was associated with 1.5-fold and 2-fold increases in odds of at least one admission for DKA in pediatric and adult patients, respectively; again, DKA readmissions was not an outcome in those studies and no further classification of the non-private insurance group was reported. ${ }^{12}{ }^{22}$ Interestingly, in our study, patients with Medicare or Medicaid were at higher risk of readmission than those who likely had no insurance coverage (self-pay). Similar patterns have been noted in other patient populations. In a mixed type 1 and type 2 diabetes population, Mays $e t$ al found that patients with recurrent DKA admission (eg, $\geq 4$ admissions) were more likely to have Medicare (25.6\%) and Medicaid (29.4\%) insurance than self-pay (10.9\%). Hasegawa $e t$ al showed that in chronic obstructive pulmonary disease (COPD), when compared with patients with private insurance, Medicaid patients had the highest risk of repeated emergency department (ED) visits (OR 1.77 and 2.92 for $>2$ and $>3$ ED visits, respectively). Patients with Medicare had only a slightly lower odds of readmission than Medicaid patients, and self-pay patients had an OR of 1.11 and 1.05 of $>2$ and $>3 \mathrm{ED}$ visits. ${ }^{23}$ In both our study and this COPD study, self-pay patients had a lower odds than those with public insurance which has policy implications. Given that the uninsured population is thought to be an even higher risk population, the reason for this finding is unclear.

Other notable predictors of DKA readmissions identified in this study were age, female sex, and leaving the hospital AMA. Each 1-year increase in age was associated with a $7 \%$ decrease in the odds of four or more DKA readmissions. The inverse association of age and DKA admission has been previously described, although the effect sizes were not as marked as in this study, ${ }^{10}{ }^{24}$ likely owing to the difference in outcome as we selected for a higher risk group. Female sex has generally been shown to be a risk factor for DKA admissions. ${ }^{10}{ }^{24}$ The reasons for this are not readily apparent. Several possible explanations include (1) men are less likely to access medical services,
(2) women may intentionally avoid glycemic control for weight gain, and 3) hormonal effects on glucose regulation. Lastly, the caregiver role of many women can sometime inadvertently result in self-neglect. ${ }^{25}$

In contrast to the pediatric population, considerably less attention has been devoted to DKA readmissions in adults with type 1 diabetes, especially in the USA. ${ }^{24-6926-29}$ While recurrent DKA admissions has been thought to be a phenomenon that typically resolves by adulthood, ${ }^{27}$ we demonstrated that $20 \%$ of adults with type 1 diabetes had at least one DKA readmission in a given calendar year. Despite the fact that recurrent DKA admissions occur more often in younger adults, mortality in a Chicago study was reported to be $16 \%$ in $\geq 4$ DKA admissions at 6-year follow-up ${ }^{2}$ and in a UK study mortality was found to be $23 \%$ in those with $\geq 5$ DKA admissions at a median 2.4-year follow-up. ${ }^{1}$ Given the staggering mortality statistics and implications of long-term complications from chronically poor glycemic control, identifying and characterizing this high-risk population of patients and intervening may improve clinical outcomes, limit acute care utilization, and reduce healthcare expenditures.

A main strength of this study was the use of a very large sample dataset that is representative at a national level of adults with type 1 diabetes. Hospital records were used to determine DKA admissions, serving as a more reliable source of DKA admission than self-report. The main limitation of this study was that we were only able to follow readmissions within a calendar year given the design of the NRD; thus, this study likely underestimates the number of readmissions in patients who were admitted in the latter portions of the calendar year and may mask seasonal variation in readmission trends. Furthermore, the lack of a consistent patient identifier across calendar years precluded our ability to report unique patients admitted across the study period. The NRD did not include information on race; thus, we were unable to evaluate interactions between race and SES. This is particularly important since ketosis-prone diabetes is more prevalent in ethnic minorities. Although these patients are often phenotyped as type 2 diabetes, it is possible that they may be misclassified as type 1 diabetes, which may result in bias, as their risk for recurrent DKA may be different. The absence of medications and laboratory data in the NRD precluded evaluation of severity of DKA, precipitating causes, and management approaches and their role in readmissions. Lastly, given the large database, some findings may be statistically significant but not necessarily be clinically relevant. Although it may be difficult to qualitatively describe the clinical impact of an individual variable, at a population level differences in proportions on the order of $5 \%$ or more may be relevant or at least draw attention to the need for further investigation.

In conclusion, lower median income and Medicare and Medicaid insurance were strong predictors of recurrent DKA admissions in adults with type 1 diabetes. Further studies are needed to understand how these 
socioeconomic factors mediate this association and to identify strategies to overcome disparities in this potentially life-threatening outcome.

Contributors EE and NNM designed the study, analyzed and interpreted the data, and wrote the manuscript.

Funding EE was supported by the Clinical Research and Epidemiology in Diabetes and Endocrinology Training Grant of the NIDDK through grant number T32 DK062707.

Competing interests None declared.

Patient consent for publication Not required.

Provenance and peer review Not commissioned; externally peer reviewed.

Data availability statement Data are available in a public, open-access repository.

Open access This is an open access article distributed in accordance with the Creative Commons Attribution Non Commercial (CC BY-NC 4.0) license, which permits others to distribute, remix, adapt, build upon this work non-commercially, and license their derivative works on different terms, provided the original work is properly cited, appropriate credit is given, any changes made indicated, and the use is non-commercial. See: http://creativecommons.org/licenses/by-nc/4.0/.

\section{REFERENCES}

1. Gibb FW, Teoh WL, Graham J, et al. Risk of death following admission to a UK hospital with diabetic ketoacidosis. Diabetologia 2016;59:2082-7.

2. Mays JA, Jackson KL, Derby TA, et al. An evaluation of recurrent diabetic ketoacidosis, fragmentation of care, and mortality across Chicago, Illinois. Diabetes Care 2016;39:1671-6.

3. Kitabchi AE, Umpierrez GE, Miles JM, et al. Hyperglycemic crises in adult patients with diabetes. Diabetes Care 2009;32:1335-43.

4. Lewis KR, Clark C, Velarde MC. Socioeconomic factors associated with pediatric diabetic ketoacidosis admissions in southern West Virginia. Clin Endocrinol 2014;81:218-21.

5. Maahs DM, Hermann JM, Holman N, et al. Rates of diabetic ketoacidosis: international comparison with 49,859 pediatric patients with type 1 diabetes from England, Wales, the U.S., Austria, and Germany. Diabetes Care 2015;38:1876-82.

6. Malik FS, Hall M, Mangione-Smith R, et al. Patient characteristics associated with differences in admission frequency for diabetic ketoacidosis in United States children's hospitals. J Pediatr 2016;171:104-10.

7. Healy-Collier K, Jones WJ, Shmerling JE, et al. Medicaid managed care reduces readmissions for youths with type 1 diabetes. Am J Manag Care 2016;22:250-6.

8. Smaldone A, Honig J, Stone PW, et al. Characteristics of California children with single versus multiple diabetic ketoacidosis hospitalizations (1998-2000). Diabetes Care 2005;28:2082-4.

9. Randall L, Begovic J, Hudson M, et al. Recurrent diabetic ketoacidosis in inner-city minority patients: behavioral, socioeconomic, and psychosocial factors. Diabetes Care 2011;34:1891-6.

10. Govan L, Maietti E, Torsney B, et al. The effect of deprivation and $\mathrm{HbA1c}$ on admission to hospital for diabetic ketoacidosis in type 1 diabetes. Diabetologia 2012;55:2356-60.

11. Lohiya S, Kreisberg R, Lohiya V. Recurrent diabetic ketoacidosis in two community teaching hospitals. Endocr Pract 2013;19:829-33.

12. Weinstock RS, Xing D, Maahs DM, et al. Severe hypoglycemia and diabetic ketoacidosis in adults with type 1 diabetes: results from the T1D exchange clinic registry. J Clin Endocrinol Metab 2013;98:3411-9.

13. Eby E, Hardwick C, Yu M, et al. Predictors of 30 day hospital readmission in patients with type 2 diabetes: a retrospective, casecontrol, database study. Curr Med Res Opin 2015;31:107-14.

14. Oakes JM, Kaufman JS. Methods in social epidemiology. 1st ed. San Francisco, CA: Jossey-Bass, 2006.

15. Agrawal S, Baird GL, Quintos JB, et al. Pediatric diabetic ketoacidosis with hyperosmolarity: clinical characteristics and outcomes. Endocr Pract 2018;24:726-32.

16. Bagdure D, Rewers A, Campagna E, et al. Epidemiology of hyperglycemic hyperosmolar syndrome in children hospitalized in USA. Pediatr Diabetes 2013;14:18-24.

17. Wolfsdorf JI, Allgrove J, Craig ME, et al. Diabetic ketoacidosis and hyperglycemic hyperosmolar state. Pediatr Diabetes 2014;15:154-79.

18. Rosenbloom AL. Hyperglycemic hyperosmolar state: an emerging pediatric problem. J Pediatr 2010;156:180-4.

19. Wachtel TJ, Tetu-Mouradjian LM, Goldman DL, et al. Hyperosmolarity and acidosis in diabetes mellitus: a three-year experience in Rhode Island. J Gen Intern Med 1991;6:495-502.

20. Zhang Z. Variable selection with stepwise and best subset approaches. Ann Trans/ Med 2016;4.

21. O'brien RM. A caution regarding rules of thumb for variance inflation factors. Qual Quant 2007:41:673-90.

22. Cengiz E, Xing D, Wong JC, et al. Severe hypoglycemia and diabetic ketoacidosis among youth with type 1 diabetes in the T1D exchange clinic registry. Pediatr Diabetes 2013;14:447-54.

23. Hasegawa K, Tsugawa Y, Tsai C-L, et al. Frequent utilization of the emergency department for acute exacerbation of chronic obstructive pulmonary disease. Respir Res 2014;15.

24. Butalia S, Johnson JA, Ghali WA, et al. Clinical and sociodemographic factors associated with diabetic ketoacidosis hospitalization in adults with type 1 diabetes. Diabet Med 2013;30:567-73.

25. Ortiz N, Lamdan R, Johnson S, et al. Caregiver status: a potential risk factor for extreme self-neglect. Psychosomatics 2009;50:166-8.

26. Howe CJ, Lewis KR. Social determinants for recurrent diabetic ketoacidosis in children with diabetes. J Pediatr Nurs 2012;27:773-4

27. Skinner TC. Recurrent diabetic ketoacidosis: causes, prevention and management. Horm Res Paediatr 2002;57:78-80.

28. Bui TP, Werther GA, Cameron FJ. Trends in diabetic ketoacidosis in childhood and adolescence: a 15-yr experience. Pediatr Diabetes 2002;3:82-8

29. Lokulo-Sodipe K, Moon RJ, Edge JA, et al. Identifying targets to reduce the incidence of diabetic ketoacidosis at diagnosis of type 1 diabetes in the UK. Arch Dis Child 2014;99:438-42. 